Review Article

\title{
Analysis of the Responsiveness of Latanoprost, Travoprost, Bimatoprost, and Tafluprost in the Treatment of OAG/OHT Patients
}

\author{
Ziyan Cai ${ }^{D},{ }^{1}$ Mengdan Cao, ${ }^{1}$ Ke Liu, ${ }^{1}$ and Xuanchu Duan ${ }^{2}{ }^{2}$ \\ ${ }^{1}$ Department of Ophthalmology, The Second Xiangya Hospital of Central South University, Changsha, Hunan, China \\ ${ }^{2}$ Department of Ophthalmology, Changsha Aier Eye Hospital, Changsha, Hunan, China \\ Correspondence should be addressed to Xuanchu Duan; duanxchu@csu.edu.cn
}

Received 22 February 2021; Accepted 18 May 2021; Published 25 May 2021

Academic Editor: Enrique Mencía-Gutiérrez

Copyright (c) 2021 Ziyan Cai et al. This is an open access article distributed under the Creative Commons Attribution License, which permits unrestricted use, distribution, and reproduction in any medium, provided the original work is properly cited.

\begin{abstract}
Aim. Within the clinical setting, some patients have been identified as lacking in response to PGAs. This meta-analysis study aimed to evaluate the responsiveness of latanoprost, travoprost, bimatoprost, and tafluprost in OAG/OHT patients, latanoprost nonresponders (LNRs), and the IOP-reducing efficacy and safety. Methods. A literature search was conducted on PubMed, Embase, and the Cochrane Controlled Trials Register. The primary clinical endpoint was the number of responders at the end of the study. The secondary clinical endpoint was the IOP reduction at the endpoint from baseline. Safety evaluation included five common adverse events: conjunctival hyperemia, hypertrichosis, ocular burning, ocular itching, and foreign-body sensation. Results. Eleven articles containing ten RCTs were included in this meta-analysis study. The results highlighted that, in the OAG/ OHT population, there was no statistically significant difference in the responsiveness of the four PGAs. Bimatoprost had a better IOP-reducing efficacy than latanoprost. There was no significant difference in the IOP-reducing efficacy of travoprost, latanoprost, and tafluprost. In LNRs, the responsiveness of bimatoprost, travoprost, and latanoprost did not show statistical differences. Bimatoprost reduced IOP with a greater extent than latanoprost and travoprost in LNRs, while there was no significant difference in the IOP-reducing efficacy of travoprost and latanoprost. No serious adverse events occurred with the treatment of the four PGAs. The prevalence of conjunctival hyperemia due to bimatoprost or tafluprost was significantly higher than that of latanoprost. Other adverse events had no significant difference between the four drugs. Conclusion. The existing studies cannot prove that latanoprost, travoprost, bimatoprost, and tafluprost have different responsiveness in OAG/OHT patients. Switching to bimatoprost or travoprost cannot achieve a significant improvement in responsiveness in LNRs. Bimatoprost has a better IOP-reducing efficacy than latanoprost and travoprost. No serious adverse events occurred during treatment with any medication we studied.
\end{abstract}

\section{Introduction}

Glaucoma is the most common irreversible blindness-inducing disease on a global scale, characterized by a chronic and progressive optic neuropathy and visual field loss [1]. Intraocular pressure (IOP) is the main risk factor for optic nerve damage, and regulation of IOP is the only clinically proven treatment that can delay disease progression [2]. Previous research has demonstrated that lowering of the IOP by $1 \mathrm{mmHg}$ can reduce the risk of glaucomatous progression by approximately $10 \%$ [3]. Topical use of ocular hypotensive agents is typically the first therapeutic option in glaucoma. Since the 1990s, prostaglandin
F2 $\alpha$ analogues (PGAs) have gradually replaced $\beta$-blockers as the first-choice therapy due to their high clinical efficacy to reduce IOP, minimal side effects, and once-daily dosage regimens, consequently facilitating patient compliance $[4,5]$.

PGAs mainly decrease IOP by increasing outflow facility through an IOP-independent uveoscleral pathway. Some studies have revealed that they can also affect the IOP-dependent conventional trabecular meshwork (TM) outflow pathway $[5,6]$. Among the PGAs used in the clinic, latanoprost and travoprost are ester prodrug analogs of prostaglandin F2 $\alpha$ (PGF2 $\alpha$ ). Though usually classified as prostaglandin analogues, bimatoprost is an amide prodrug 
of 17 -phenyl-PGF $2 \alpha$ (similar to PGF2 $\alpha$ ) $[1,7]$. Tafluprost is a unique PGF $2 \alpha$ analog. The major modification of tafluprost is the substitution of the $\mathrm{C}-15$ hydrogen and hydroxyl group with two fluorine atoms [8]. PGAs can bind to prostaglandin receptors EP and FP in the ciliary muscle, induce ciliary muscle relaxation, and increase uveoscleral outflow facility. These drugs also increase cell contractility of the TM as well as decrease endothelial cell contractility within Schlemm's canal, mediating aqueous humor outflow through the conventional pathway [9]. PGAs can degrade the extracellular matrix (ECM), which results in ECM turnover in the uvea and TM and ultimately reduces outflow resistance [10].

PGAs have been proven effective in decreasing IOP and are widely used in the treatment of glaucoma. However, after PGA therapies, some patients do not demonstrate a significant reduction in IOP,or fail to achieve the target IOP. Such patients are usually defined as nonresponders. Although there is no clear definition, nonresponders typically refer to patients with an IOP reduction of $<15 \%$ from baseline after treatment [11]. It was reported that, in American and European populations, 12-41\% of patients with glaucoma demonstrated low response to latanoprost, and such patients are defined as latanoprost nonresponders (LNRs). In Singapore, these data are approximately 5.4\% [12]. Martínez and colleagues [13] reported that approximately $11 \%$ of Hispanics are LNRs. It was reported that the efficacy of latanoprost is significantly undermined in elderly patients and in European and American populations [4]. However, other studies revealed that age and baseline IOP are not factors affecting patient responsiveness to PGAs [14, 15]. Several studies demonstrated that replacing latanoprost with bimatoprost or travoprost can further decrease IOP in LNRs [16-18]. This can be due to varying PGA not acting on the same receptor [19]. Some researchers postulated that bimatoprost interacts with a unique receptor that is unassociated with other PGA receptors. However, this "undefined receptor" has not yet been cloned $[1,10]$.

This meta-analysis aimed to evaluate the responsiveness of latanoprost $(0.005 \mathrm{mg} / \mathrm{mL})$, travoprost $(0.004 \mathrm{mg} / \mathrm{mL})$, bimatoprost $(0.03 \mathrm{mg} / \mathrm{mL})$, and tafluprost $(0.0015 \mathrm{mg} / \mathrm{mL})$ eye-dropbased therapies in patients with open-angle glaucoma (OAG) or ocular hypertension (OHT) and whether substituting latanoprost with other PGAs can further reduce the IOP in LNRs. This study also evaluated the IOP-reducing efficacy and safety of the four PGAs in patients with OAG or OHT.

\section{Materials and Methods}

2.1. Literature Search Strategy. A thorough literature search was carried out separately by two researchers on PubMed, Embase, and the Cochrane Controlled Trials Register. The publication time of the selected articles was before 1990, with the type of study restricted to randomized controlled trials (RCTs). The keyword input strategy was "open angle glaucoma" OR "ocular hypertension" AND "latanoprost" OR "travoprost" OR "bimatoprost" OR "tafluprost." Manual examination of reference lists was carried out, for relevant original research to supplement the study. Following reading the title and abstract, whenever a research study article was deemed relevant to this research, the full text was read.

\subsection{Inclusion Criteria}

(a) Research type: RCT

(b) Research object: patients with mild to moderate OAG/OHT and IOP between 21 and $39 \mathrm{mmHg}$ after drug washing

(c) Research content: comparing the responsiveness of latanoprost, travoprost, bimatoprost, and tafluprost in OAG/OHT patients or LNRs

(d) The subjects did not undergo any eye surgery within 1 year before treatment

(e) Follow-up time $\geq 1$ month

\subsection{Exclusion Criteria}

(a) Nonclinical research, animal research, retrospective research, case report, and review

(b) Less than 10 patients in each study group

(c) Repeated data

(d) Severe OAG with uncontrollable IOP, angle-closure glaucoma, neovascular glaucoma, uveitic glaucoma, and patients who have undergone any eye surgery within one year before participating in the research

(e) Follow-up time $<1$ month

(f) Drugs other than the four drugs studied in this metaanalysis were added to the research plan or combined with other antihypertensive treatments

2.4. Data Extraction and Clinical Endpoints. Two researchers extracted metadata separately. Any disagreements were submitted to the third senior researcher for adjudication. The data extracted from the trials included the first author, publication year, blind status, research design (parallel group or crossover study), treatment, single center or multicenter, sample size, type of glaucoma, age, sex, ethnicity, duration of follow-up, number of patients lost to follow-up, and LNR status.

The primary clinical endpoint was the number of responders at the end of each study. A responder was defined as a patient with an IOP reduction of $\geq 15 \%$ or $\geq 20 \%$ from baseline, after therapy. When both the data of IOP reduction $\geq 15 \%$ or $20 \%$ were described in a study, the data of IOP reduction $\geq 20 \%$ were extracted. The secondary clinical endpoint was the IOP reduction at the endpoint from baseline. Safety evaluation included five common adverse events: conjunctival hyperemia, hypertrichosis, ocular burning, ocular itching, and foreign-body sensation.

2.5. Quality Assessment. Two researchers blindly evaluated the quality of the studies reported in the scientific literature with the Methodological Quality Assessment (Modified Jadad Score). If the results differed, it was resolved through discussion with a third senior researcher. The quality assessment included four items: random sequence generation 
(appropriate: 2, unclear: 1, and inappropriate: 0), concealment of allocation (appropriate: 2, unclear: 1, and inappropriate: 0 ), blind (double blind: 2 , single blind: 1 , and open label: 0 ), and withdrawals and dropouts (described: 1 and not described: 0 ). If the Jadad score of a document was $\leq 3$, we defined the study as "low quality." If the score was between 4 and 7 , we defined the research as "high quality."
2.6. Data Analysis. The Stata SE-64 ${ }^{\circledR}$ software (StataCorp, College Station, TX, USA) was used for data analysis. We used the relative risk (RR) to summarize dichotomous results and the weighted mean difference (WMD) to summarize continuous results. When the IOP reduction and the standard deviation (SD) value were not available from the original document, we utilized the following formula to calculate [20]:

$$
\begin{aligned}
\Delta \mathrm{IOP} & =\mathrm{IOP}_{\text {baseline }}-\mathrm{IOP}_{\text {end point }} \\
\mathrm{SD}_{\Delta \mathrm{IOP}} & =\sqrt{(\mathrm{SD} \text { baseline } 2+\mathrm{SD} \text { end point } 2-\mathrm{SD} \text { baseline } \times \mathrm{SD} \text { end point })} .
\end{aligned}
$$

The heterogeneity was analyzed by $\mathrm{I}^{2}$ statistics. $P<0.05$ was defined as an index of heterogeneity. When $\mathrm{I}^{2}>50 \%$, we considered that heterogeneity was confirmed and we chose a random-effects model for pooling the data in such a situation. When $\mathrm{I}^{2} \leq 50 \%$, we chose a fixed-effect model. Due to the limitation of the number of included studies, we did not perform sensitivity analysis and publication bias evaluation.

\section{Results}

3.1. Study Selection. A total of 889 documents were retrieved and 588 documents remained (following removal of duplicates), of which 236 documents were initially selected following reading the title and abstract. According to the applied inclusion and exclusion criteria, we selected 14 documents deemed as relevant and censored the full text, including references. Two articles were excluded as the research content did not match with this study. One article was excluded due to incomplete data. Finally, 11 articles containing 10 RCTs were included in this meta-analysis $[8,11,16-18,21-26]$. The detailed search strategy is outlined in Figure 1. Choplin [11] and Noecker [21] studied the same population, though the authors extracted differing outcome variables. A total of 1381 patients were included, with an average age of 60.1 years, of which $45.1 \%$ were men. The follow-up time range was from one to six months. Race included white, black, Hispanic, Asian, and Native Hawaiian. The included studies did not analyze patient compliance issues. However, since all the included studies were prospective RCTs and the patient data lost to follow-up were excluded, the analytical assumption placed in this study was that there existed full patient compliance. The detailed data of each study are highlighted in Table 1.

3.2. Quality Assessment. According to the revised Jadad scale, two of the studies were defined as "low quality" and the other eight studies were defined as "high quality." The average score of the included studies was five points. Due to the limitation of the number of included studies, we did not employ a funnel plot to assess publication bias. The specific score of each study is highlighted in Table 2.

\subsection{Responsiveness and Efficacy of PGAs in $O A G / O H T$}

3.3.1. Responsiveness of PGAs in OAG/OHT. Two trials compared the responsiveness of bimatoprost and latanoprost in OAG/OHT. One trial compared bimatoprost and travoprost in OAG/OHT. Heterogeneity test results across the bimatoprost group and latanoprost group were chi square $=19.23, P \leq 0.001$, and $\mathrm{I}^{2}=94.8 \%$. We used the randomized effect model for analysis. Three trials compared the responsiveness of tafluprost and latanoprost, and one trial compared tafluprost and travoprost in OAG/OHT. Heterogeneity test results across the tafluprost and latanoprost study groups were chi square $=2.32, P=0.314$, and $\mathrm{I}^{2}=13.7 \%$. The fixed-effect model was employed for analysis. The results were expressed by RR and $95 \%$ confidence interval. The responsiveness of bimatoprost was higher than that of latanoprost and travoprost, though the difference was not statistically significant (bimatoprost vs. latanoprost: $\mathrm{RR}=1.301,95 \% \mathrm{CI}$ : 0.711-2.380, $P=0.394$; bimatoprost vs. travoprost: $\mathrm{RR}=1.208,95 \% \mathrm{CI}: 0.964-1.514, P=0.101$ ). The responsiveness of tafluprost was higher than that of latanoprost and travoprost, while the variation was also not statistically significant (tafluprost vs. latanoprost: $\mathrm{RR}=1.101$, 95\% CI: $0.973-1.245, P=0.127$; tafluprost vs. travoprost: $\mathrm{RR}=1.081,95 \% \mathrm{CI}: 0.771-1.516, P=0.652)$. Detailed data are listed in Table 3.

3.3.2. Efficacy of $P G A s$ in $O A G / O H T$. One trial compared the IOP-decreasing effect of bimatoprost and latanoprost in OAG/OHT. Three trials compared the IOP-reducing effect of tafluprost and latanoprost; one trial compared the IOPreducing effect of tafluprost and travoprost in OAG/OHT. Reduction of IOP from baseline is listed in Table 4. Heterogeneity test results across tafluprost and latanoprost study groups were chi square $=0.19, \quad P=0.909$, and $\mathrm{I}^{2}=0.0 \%$. The fixed-effect model was employed for analysis. The results were expressed by WMD and $95 \%$ confidence interval. Efficacy outcome revealed a statistically significant difference between bimatoprost and latanoprost (bimatoprost vs. latanoprost: $\mathrm{WMD}=1.000,95 \% \mathrm{CI}$ : $0.130-1.870$, $P=0.024$ ), suggesting that bimatoprost was more effective than latanoprost in lowering IOP. There was no significant difference in the IOP-reducing efficacy of travoprost, 


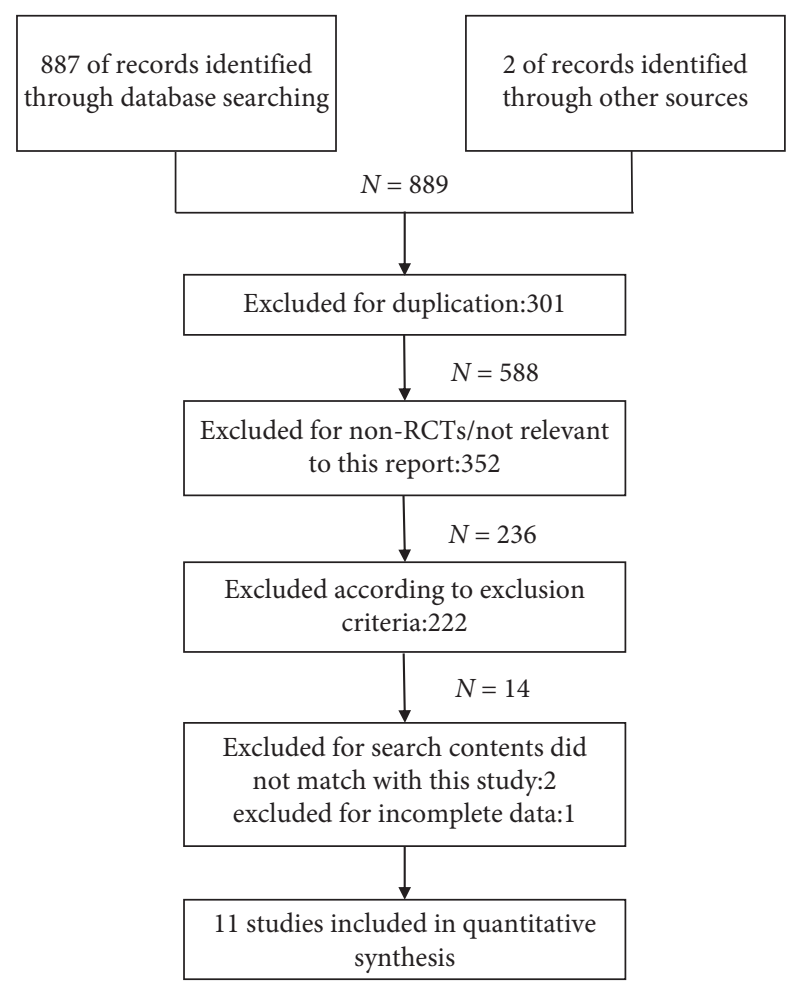

Figure 1: Flow chart of the literature search process.

latanoprost, and tafluprost (tafluprost vs. travoprost: $\mathrm{WMD}=0.100,95 \% \mathrm{CI}$ : $-1.414-1.614, P=0.897$; tafluprost vs. latanoprost: $\mathrm{WMD}=0.534,95 \% \mathrm{CI}:-0.168-1.236$, $P=0.136)$. Detailed data are listed in Table 5. Due to lack of original data, we did not compare the efficacy of bimatoprost and travoprost, the efficacy of travoprost and latanoprost, or the efficacy of tafluprost and bimatoprost in OAG/OHT.

\subsection{Responsiveness and Efficacy of PGAs in LNRs}

3.4.1. Responsiveness of PGAs in LNRs. Two trials compared the responsiveness of bimatoprost and latanoprost in LNRs (heterogeneity test: chi square $=6.54, P=0.011, \mathrm{I}^{2}=84.7 \%$ ). Two trials compared the responsiveness of bimatoprost and travoprost in LNRs (heterogeneity test: chi square $=2.49$, $\left.P=0.115, \mathrm{I}^{2}=59.8 \%\right)$. These two analyses employed the randomized effect model. One trial compared the responsiveness of travoprost and latanoprost in LNRs. Responsiveness outcome showed no significant difference between the three PGAs (bimatoprost vs. latanoprost: $\mathrm{RR}=4.934$, 95\% CI:0.139-175.638, $P=0.381$; bimatoprost vs. travoprost: $\mathrm{RR}=1.361,95 \% \mathrm{CI}: 0.703-2.635, P=0.360$; travoprost vs. latanoprost: $\mathrm{RR}=1.401,95 \%$ CI:0.685-2.864, $P=0.355)$. Detailed data are listed in Table 6. Due to lack of original data, the responsiveness of tafluprost could not be evaluated in LNRs.

3.4.2. Efficacy of PGAs in LNRs. Two trials compared the efficacy of bimatoprost and latanoprost in LNRs (heterogeneity test: chi square $\left.=9.63, P=0.002, \mathrm{I}^{2}=89.6 \%\right)$. This analysis employed the randomized effect model. Two trials compared the efficacy of bimatoprost and travoprost in LNRs (heterogeneity test: chi square $=0.01, P=0.944$, $\mathrm{I}^{2}=0.0 \%$ ). In this case, the fixed-effect model was utilized. One trial compared the efficacy of travoprost and latanoprost in LNRs. Reduction of IOP from baseline is listed in Table 7. Efficacy outcome revealed that bimatoprost provided a greater reduction in IOP than latanoprost and travoprost, though there was no statistically significant difference in drug efficacy between travoprost and latanoprost (bimatoprost vs. latanoprost: $\mathrm{WMD}=4.153,95 \%$ CI: $0.245-8.062, P=0.037$; bimatoprost vs. travoprost: $\mathrm{WMD}=0.695,95 \% \mathrm{CI}: 0.114-1.277, P=0.019$; travoprost vs. latanoprost: $\mathrm{WMD}=1.400,95 \% \mathrm{CI}:-1.360-4.160$, $P=0.320)$. Detailed data are listed in Table 8 .

3.5. Safety. No serious adverse reactions occurred on patient treatment with the four PGAs. The most common adverse event was conjunctival hyperemia. The prevalence of conjunctival hyperemia was significantly higher in bimatoprost and tafluprost than latanoprost (bimatoprost vs. latanoprost: $\mathrm{RR}=2.556,95 \% \mathrm{CI}: 1.844-3.542, P \leq 0.001$; tafluprost vs. latanoprost: $\mathrm{RR}=1.779,95 \% \mathrm{CI}: 1.057-2.995, P=0.030)$. The prevalence of conjunctival hyperemia was also higher in bimatoprost than travoprost though the difference was not statistically significant (bimatoprost vs. travoprost: $\mathrm{RR}=1.471,95 \% \mathrm{CI}: 0.676-3.200, P=0.330)$. There was no statistically significant difference in the prevalence of burning-eye sensation between bimatoprost and latanoprost and tafluprost and latanoprost (bimatoprost vs. latanoprost: $\mathrm{RR}=1.169$, 95\%CI: $0.436-3.132, P=0.757$; tafluprost vs. latanoprost: $\mathrm{RR}=0.990,95 \% \mathrm{CI}: 0.151-6.477, P=0.991)$ and no statistically significant difference in the prevalence of foreign-body sensation between bimatoprost and travoprost and tafluprost and latanoprost (bimatoprost vs. travoprost: $\mathrm{RR}=0.325,95 \% \mathrm{CI}: 0.034-3.080, P=0.327$; tafluprost vs. latanoprost: $\mathrm{RR}=0.497,95 \% \mathrm{CI}: 0.174-1.425, P=0.193)$. In addition, there was no statistically significant difference in the prevalence of hypertrichosis (bimatoprost vs. latanoprost: $\mathrm{RR}=0.562,95 \% \mathrm{CI}: 0.002-160.502, P=0.842$; bimatoprost vs. travoprost: $\mathrm{RR}=5.152,95 \% \mathrm{CI}$ : $0.250-106.293$, $P=0.288$ ) and itching (bimatoprost vs. latanoprost: $\mathrm{RR}=0.345$, 95\%CI: 0.115-1.031, $P=0.057$; bimatoprost vs. travoprost: $\mathrm{RR}=1.304,95 \% \mathrm{CI}: 0.301-5.641, P=0.723)$ in bimatoprost, travoprost, and latanoprost. There was no statistically significant difference in the prevalence of itching between tafluprost and latanoprost (tafluprost vs. latanoprost: $\mathrm{RR}=0.985,95 \% \mathrm{CI}: 0.349-2.775, P=0.977)$. Detailed data are listed in Table 9.

\section{Discussion}

Presently, five categories of antihypertensive drugs are clinically used to treat glaucoma: $\beta$-blockers, carbonic anhydrase inhibitors (CAIs), sympathomimetics, $\alpha-2$ adrenergic agonists, and PGAs [1, 27]. PGAs have proven to be more effective than the other four drug classes in reducing IOP and are widely used as first-line of treatment for 
TABLE 1: Characteristics of studies included in the meta-analysis.

\begin{tabular}{|c|c|c|c|c|c|c|c|c|c|c|c|c|c|}
\hline Author & Year & Blind & Design & Center & Treatment & $\begin{array}{c}\text { Number } \\
\text { of } \\
\text { patients }\end{array}$ & $\begin{array}{c}\text { Age } \\
\text { (years) }\end{array}$ & $\begin{array}{l}\text { Type of } \\
\text { diagnosis }\end{array}$ & LNR & $\begin{array}{l}\text { Male/ } \\
\text { female }\end{array}$ & Race & $\begin{array}{l}\text { Follow- } \\
\text { up } \\
\text { (months) }\end{array}$ & $\begin{array}{l}\text { Lost to } \\
\text { follow- } \\
\text { up }\end{array}$ \\
\hline $\begin{array}{l}\text { Blondeau } \\
\text { et al. [17] }\end{array}$ & 2019 & No & PG & Single & $\begin{array}{l}\text { LAT vs. } \\
\text { TRA vs. } \\
\text { BIM }\end{array}$ & 83 & 68.8 & $\begin{array}{l}\text { OAG } \\
\text { and } \\
\text { OHT }\end{array}$ & Yes & $43 / 40$ & White & 1 & 0 \\
\hline $\begin{array}{l}\text { Choplin et al. } \\
\text { [11] }\end{array}$ & 2004 & Single & PG & Multi & $\begin{array}{l}\text { BIM vs. } \\
\text { LAT }\end{array}$ & 269 & 61.3 & $\begin{array}{l}\text { OAG } \\
\text { and } \\
\text { OHT }\end{array}$ & No & $\begin{array}{c}103 / \\
169\end{array}$ & $\begin{array}{c}\text { White } \\
(83 \%), \\
\text { black } \\
(11 \%) \text {, and } \\
\text { Hispanic } \\
(6 \%)\end{array}$ & 6 & $\begin{array}{c}20 / 269 \\
(7.43 \%)\end{array}$ \\
\hline $\begin{array}{l}\text { Noecker } \\
\text { et al. [21] }\end{array}$ & 2003 & Single & PG & Multi & $\begin{array}{l}\text { BIM vs. } \\
\text { LAT }\end{array}$ & 269 & 61.3 & $\begin{array}{l}\text { OAG } \\
\text { and } \\
\text { OHT }\end{array}$ & No & $\begin{array}{c}103 / \\
169\end{array}$ & $\begin{array}{c}\text { White } \\
(83 \%), \\
\text { black } \\
(11 \%) \text {, and } \\
\text { Hispanic } \\
(6 \%)\end{array}$ & 6 & $\begin{array}{c}20 / 269 \\
(7.43 \%)\end{array}$ \\
\hline $\begin{array}{l}\text { Kammer } \\
\text { et al. [16] }\end{array}$ & 2010 & Single & PG & Multi & $\begin{array}{c}\text { BIM vs. } \\
\text { TRA }\end{array}$ & 266 & 63.0 & $\begin{array}{l}\text { OAG } \\
\text { and } \\
\text { OHT }\end{array}$ & Yes & $\begin{array}{l}119 / \\
147\end{array}$ & $\begin{array}{c}\text { White } \\
(57.3 \%), \\
\text { black } \\
(27.5 \%), \\
\text { Hispanic } \\
(12.2 \%), \\
\text { asian } \\
(1.5 \%) \text {, and } \\
\text { native } \\
\text { Hawaiian } \\
(1.5 \%)\end{array}$ & 3 & $\begin{array}{c}7 / 266 \\
(2.63 \%)\end{array}$ \\
\hline $\begin{array}{l}\text { Konstas et al. } \\
\text { [22] }\end{array}$ & 2007 & Single & CR & Multi & $\begin{array}{l}\text { BIM vs. } \\
\text { LAT }\end{array}$ & 129 & 66.5 & XFG & No & N. A & White & $3 \times 2$ & $\begin{array}{c}6 / 129 \\
(4.66 \%)\end{array}$ \\
\hline $\begin{array}{l}\text { Noecker } \\
\text { et al. [23] }\end{array}$ & 2006 & Single & PG & Multi & $\begin{array}{l}\text { BIMvs. } \\
\text { TRA }\end{array}$ & 94 & 63.4 & $\begin{array}{l}\text { OAG } \\
\text { and } \\
\text { OHT }\end{array}$ & No & $37 / 57$ & Black & 3 & $\begin{array}{c}3 / 94 \\
(3.20 \%)\end{array}$ \\
\hline $\begin{array}{l}\text { Gandolfi and } \\
\text { Cimino [18] }\end{array}$ & 2003 & Double & CR & Single & $\begin{array}{l}\text { BIM vs. } \\
\text { LAT }\end{array}$ & 15 & 62.0 & $\begin{array}{l}\text { OAG } \\
\text { and } \\
\text { OHT }\end{array}$ & Yes & $6 / 9$ & N. A & $1 \times 2$ & 0 \\
\hline $\begin{array}{l}\text { Kuwayama } \\
\text { and } \\
\text { Komemushi } \\
{[24]}\end{array}$ & 2008 & Single & PG & Multi & $\begin{array}{l}\text { TAF vs. } \\
\text { LAT }\end{array}$ & 125 & 59.0 & $\begin{array}{l}\text { OAG } \\
\text { and } \\
\text { OHT }\end{array}$ & No & $55 / 42$ & Asian & 1 & $\begin{array}{l}20 / 125 \\
(16.0 \%)\end{array}$ \\
\hline $\begin{array}{l}\text { Traverso } \\
\text { et al. [25] }\end{array}$ & 2010 & Double & PG & Multi & $\begin{array}{c}\text { TAF vs. } \\
\text { LAT }\end{array}$ & 38 & N. A & OAG & No & $12 / 26$ & White & 1.5 & $\begin{array}{c}2 \\
(5.26 \%)\end{array}$ \\
\hline Ge et al. [8] & 2015 & Single & PG & Multi & $\begin{array}{l}\text { TAF vs. } \\
\text { LAT }\end{array}$ & 246 & 44.0 & $\begin{array}{l}\text { OAG } \\
\text { and } \\
\text { OHT }\end{array}$ & No & $125 / 71$ & Asian & 1 & $\begin{array}{c}21 / 246 \\
(8.53 \%)\end{array}$ \\
\hline $\begin{array}{l}\text { Mizoguch } \\
\text { et al. [26] }\end{array}$ & 2012 & No & CR & Multi & $\begin{array}{c}\text { TAF vs. } \\
\text { TRA }\end{array}$ & 116 & 69.4 & NTG & No & $23 / 67$ & Asian & $3 \times 2$ & $\begin{array}{l}20 / 116 \\
(17.2 \%) \\
\end{array}$ \\
\hline
\end{tabular}

*PG: parallel group, CR: crossover, XFG: exfoliative glaucoma, BIM: bimatoprost, TRA: travoprost, LAT: latanoprost, TAF: tafluprost.

glaucoma $[4,27,28]$. However, previous studies did not find significant differences in the efficacy of PGAs [16, 23, 27].

PGAs currently available on the market include latanoprost, travoprost, bimatoprost, tafluprost, and unoprostone. Latanoprost and travoprost are synthetic ester prodrugs of natural PGF2 $\alpha$. Their hydrolysis products manifest their action through binding to the FP and EP receptors in the ciliary body, causing relaxation of the ciliary muscle and increasing outflow facility of the IOP-independent uveoscleral pathway. Such by-products can also increase TM pathway outflow facility, albeit mainly through the uveoscleral pathway $[18,29-31]$. With a unique chemical structure different from other PGAs, bimatoprost is a synthetic anamide prodrug of 17-phenyl-PGF2 $\alpha$ [5]. Bimatoprost can act as a complete molecule to decrease IOP, without hydrolysis, to be biologically active as latanoprost and travoprost $[17,18]$. The hydrolysate of bimatoprost (17phenyl PGF $2 \alpha$, also named bimatoprost acid) is also an effective FP receptor agonist that can promote outflow facility by activating the FP receptor [7]. Several studies demonstrated that bimatoprost can reduce IOP through a pathway that is independent of FP receptors [32]. The major 
TABLE 2: Quality assessment of the included studies.

\begin{tabular}{|c|c|c|c|c|c|c|}
\hline & & $\begin{array}{c}\text { Random sequence } \\
\text { generation }\end{array}$ & $\begin{array}{c}\text { Concealment of } \\
\text { allocation }\end{array}$ & $\begin{array}{c}\text { Double } \\
\text { blind }\end{array}$ & $\begin{array}{c}\text { Withdrawals and } \\
\text { dropouts }\end{array}$ & $\begin{array}{l}\text { Total } \\
\text { score }\end{array}$ \\
\hline Blondeau et al. [17] & 2019 & 2 & 0 & 0 & 1 & 3 \\
\hline Noecker et al. [21] & 2003 & 2 & 2 & 1 & 1 & 6 \\
\hline Kammer et al. [16] & 2010 & 2 & 2 & 1 & 1 & 6 \\
\hline Konstas et al. [22] & 2007 & 1 & 1 & 1 & 1 & 4 \\
\hline Noecker et al. [23] & 2006 & 2 & 1 & 1 & 1 & 5 \\
\hline Gandolfi and Cimino [18] & 2003 & 1 & 1 & 1 & 1 & 4 \\
\hline $\begin{array}{l}\text { Kuwayama and Komemushi } \\
\text { [24] }\end{array}$ & 2008 & 2 & 2 & 1 & 1 & 6 \\
\hline Traverso et al. [25] & 2010 & 2 & 2 & 2 & 1 & 7 \\
\hline Ge et al. [8] & 2015 & 2 & 2 & 1 & 1 & 6 \\
\hline Mizoguch et al. [26] & 2012 & 1 & 1 & 0 & 1 & 3 \\
\hline
\end{tabular}

TABle 3: Responsiveness of PGAs in OAG/OHT.

\begin{tabular}{lcccccccc}
\hline & $\begin{array}{c}\text { Number of } \\
\text { trials }\end{array}$ & $\begin{array}{c}\text { BIM } \\
(\mathrm{N})\end{array}$ & $\begin{array}{c}\text { TRA } \\
(\mathrm{N})\end{array}$ & $\begin{array}{c}\text { LAT } \\
(\mathrm{N})\end{array}$ & $\begin{array}{c}\text { TAF } \\
(\mathrm{N})\end{array}$ & RR (95\%CI) & $\begin{array}{c}\text { Test for } \\
\text { heterogeneity }\end{array}$ & $\begin{array}{c}\text { Test for overall } \\
\text { effect }\end{array}$ \\
\hline BIM vs. LAT & 2 & 257 & 0 & 261 & 0 & $\begin{array}{c}1.301(0.711, \\
2.380)\end{array}$ & $Q=19.23, P \leq 0.001$ & $Z=0.85, P=0.394$ \\
BIM vs. TRA & 1 & 47 & 44 & 0 & 0 & $\begin{array}{c}1.208(0.964, \\
1.514)\end{array}$ & N. A & $Z=1.64, P=0.101$ \\
TAF vs. LAT & 3 & 0 & 0 & 193 & 186 & $\begin{array}{c}1.101(0.973, \\
1.245)\end{array}$ \\
TAF vs. TRA & 1 & 0 & 90 & 0 & 90 & $\begin{array}{c}1.081(0.771, \\
1.516)\end{array}$ & $Q=2.32, P=0.314$ & $Z=1.52, P=0.127$ \\
\hline
\end{tabular}

*BIM: bimatoprost, TRA: travoprost, LAT: latanoprost, TAF: tafluprost.

TABLE 4: Reduction of IOP from baseline of PGAs in OAG/OHT.

\begin{tabular}{|c|c|c|c|c|c|}
\hline Author & Year & BIM (n) & TRA (n) & LAT (n) & TAF (n) \\
\hline Konstas et al. [22] & 2007 & $9.3 \pm 3.4(124)$ & N. A & $8.3 \pm 3.6(125)$ & N. A \\
\hline Mizoguch et al. [26] & 2012 & N. A & $2.2 \pm 2.2(90)$ & N. A & $2.3 \pm 2.3(90)$ \\
\hline Kuwayama and Komemushi [24] & 2008 & N. A & N. A & $6.2 \pm 2.5(51)$ & $6.6 \pm 2.5(46)$ \\
\hline Traverso et al. [25] & 2010 & N. A & N. A & $8.6 \pm 3.0(18)$ & $9.5 \pm 3.1(18)$ \\
\hline Ge et al. [8] & 2015 & N. A & N. A & $9.2 \pm 4.1(105)$ & $9.8 \pm 4.0(91)$ \\
\hline
\end{tabular}

*BIM: bimatoprost, TRA: travoprost, LAT: latanoprost, TAF: tafluprost.

TABle 5: Efficacy of PGAs in OAG/OHT

\begin{tabular}{lcccccccc}
\hline & $\begin{array}{c}\text { Number of } \\
\text { trails }\end{array}$ & $\begin{array}{c}\text { BIM } \\
(\mathrm{N})\end{array}$ & $\begin{array}{c}\text { TRA } \\
(\mathrm{N})\end{array}$ & $\begin{array}{c}\text { LAT } \\
(\mathrm{N})\end{array}$ & $\begin{array}{c}\text { TAF } \\
(\mathrm{N})\end{array}$ & WMD (95\%CI) & $\begin{array}{c}\text { Test for } \\
\text { heterogeneity }\end{array}$ & $\begin{array}{c}\text { Test for overall } \\
\text { effect }\end{array}$ \\
\hline BIM vs. LAT & 1 & 124 & 0 & 125 & 0 & $\begin{array}{c}1.000(0.130,1.870) \\
0.100(-1.414, \\
1.614)\end{array}$ & N. A & N. A \\
TAF vs. TRA & 1 & 90 & 90 & 0 & 0 & $\begin{array}{c}0.534(-0.168, \\
1.236)\end{array}$ & $Q=0.19, P=0.909, P=0.024$ \\
TAF vs. LAT & 3 & 0 & 0 & 174 & 155 & $Z=1.49, P=0.136$ \\
\hline
\end{tabular}

*BIM: bimatoprost, TRA: travoprost, LAT: latanoprost, TAF: tafluprost.

TABLE 6: Responsiveness of PGAs in LNRs.

\begin{tabular}{lccccccc}
\hline & Number of trials & BIM (N) & TRA (N) & LAT (N) & RR (95\%CI) & Test for heterogeneity & Test for overall effect \\
\hline BIM vs. LAT & 2 & 46 & 0 & 44 & $4.934(0.139,175.638)$ & $Q=6.54, P=0.011$ & $Z=0.88, P=0.381$ \\
BIM vs. TRA & 2 & 162 & 158 & 0 & $1.361(0.703,2.635)$ & $Q=2.49, P=0.115$ & $Z=0.91, P=0.360$ \\
TRA vs. LAT & 1 & 0 & 23 & 29 & $1.401(0.685,2.864)$ & N. A & $Z=0.92, P=0.355$ \\
\hline
\end{tabular}

*BIM: bimatoprost, TRA: travoprost, LAT: latanoprost. 
TABLE 7: Reduction of IOP from baseline of PGAs in LNRs.

\begin{tabular}{lcccc}
\hline Author & Year & Bimatoprost (n) & Travoprost (n) & Latanoprost (n) \\
\hline Gandolfi and Cimino [18] & 2003 & $6.7 \pm 1.5(15)$ & N. A & $0.7 \pm 1.0(15)$ \\
Kammer et al. [16] & 2010 & $2.1 \pm 2.4(128)$ & $1.4 \pm 2.5(132)$ & N. A \\
Blondeau et al. [17] & 2019 & $4.9 \pm 4.6(31)$ & $4.3 \pm 5.3(23)$ & $2.9 \pm 4.7(29)$ \\
\hline
\end{tabular}

TABLe 8: Efficacy of PGAs in LNRs.

\begin{tabular}{lccccccc}
\hline & Number of trials & BIM (N) & TRA (N) & LAT (N) & WMD (95\%CI) & Test for heterogeneity & Test for overall effect \\
\hline BIM vs. LAT & 2 & 46 & 0 & 44 & $4.153(0.245,8.062)$ & $Q=9.63, P=0.002$ & $Z=2.08, P=0.037$ \\
BIM vs. TRA & 2 & 162 & 158 & 0 & $0.695(0.114,1.277)$ & $Q=0.01, P=0.944$ & $Z=2.34, P=0.019$ \\
TRA vs. LAT & 1 & 0 & 23 & 29 & $1.400(-1.360,4.160)$ & N. A & $Z=0.99, P=0.320$ \\
\hline
\end{tabular}

*BIM: bimatoprost, TRA: travoprost, LAT: latanoprost.

TABLE 9: Safety analysis for PGAs.

\begin{tabular}{|c|c|c|c|c|c|c|c|c|c|}
\hline & & $\begin{array}{c}\text { Number of } \\
\text { trials }\end{array}$ & $\begin{array}{c}\text { BIM } \\
(\mathrm{N})\end{array}$ & $\begin{array}{l}\text { TRA } \\
(\mathrm{N})\end{array}$ & $\begin{array}{l}\text { LAT } \\
(\mathrm{N})\end{array}$ & $\begin{array}{l}\text { TAF } \\
(\mathrm{N})\end{array}$ & RR (95\%CI) & $\begin{array}{c}\text { Test for } \\
\text { heterogeneity }\end{array}$ & $\begin{array}{c}\text { Test for overall } \\
\text { effect }\end{array}$ \\
\hline \multirow{3}{*}{$\begin{array}{l}\text { Conjunctival } \\
\text { hyperemia }\end{array}$} & $\begin{array}{l}\text { BIM vs. } \\
\text { LAT }\end{array}$ & 3 & 272 & 0 & 276 & 0 & $\begin{array}{c}2.556(1.844 \\
3.542)\end{array}$ & $\begin{array}{c}Q=1.87 \\
P=0.392\end{array}$ & $\begin{array}{l}Z=5.63 \\
P \leq 0.001\end{array}$ \\
\hline & $\begin{array}{l}\text { BIM vs. } \\
\text { TRA }\end{array}$ & 2 & 180 & 180 & 0 & 0 & $\begin{array}{c}1.471(0.676 \\
3.200)\end{array}$ & $\begin{aligned} Q & =0.22 \\
P & =0.639\end{aligned}$ & $\begin{aligned} Z & =0.97 \\
P & =0.330\end{aligned}$ \\
\hline & $\begin{array}{l}\text { TAF vs. } \\
\text { LAT }\end{array}$ & 2 & 0 & 0 & 174 & 175 & $\begin{array}{c}1.779(1.057 \\
2.995)\end{array}$ & $\begin{aligned} Q & =0.78 \\
P & =0.378\end{aligned}$ & $\begin{aligned} Z & =2.17 \\
P & =0.030\end{aligned}$ \\
\hline \multirow{2}{*}{ Burning } & $\begin{array}{c}\text { BIM vs. } \\
\text { LAT }\end{array}$ & 1 & 133 & 0 & 136 & 0 & $\begin{array}{c}1.169(0.436 \\
3.132)\end{array}$ & N.A. & $\begin{aligned} Z & =0.31 \\
P & =0.757\end{aligned}$ \\
\hline & $\begin{array}{c}\text { TAF vs. } \\
\text { LAT }\end{array}$ & 2 & 0 & 0 & 174 & 175 & $\begin{array}{c}0.990(0.151 \\
6.477)\end{array}$ & $\begin{aligned} Q & =4.87 \\
P & =0.027\end{aligned}$ & $\begin{array}{c}Z=001 \\
P=0.991\end{array}$ \\
\hline \multirow{2}{*}{$\begin{array}{l}\text { Foreign body } \\
\text { sensation }\end{array}$} & $\begin{array}{l}\text { BIM vs. } \\
\text { TRA }\end{array}$ & 2 & 180 & 180 & 0 & 0 & $\begin{array}{c}0.325(0.034 \\
3.080)\end{array}$ & $\begin{array}{c}Q=0.00 \\
P=0.961\end{array}$ & $\begin{aligned} Z & =0.98 \\
P & =0.327\end{aligned}$ \\
\hline & $\begin{array}{l}\text { TAF vs. } \\
\text { LAT }\end{array}$ & 2 & 0 & 0 & 174 & 175 & $\begin{array}{c}0.497(0.174 \\
1.425)\end{array}$ & $\begin{array}{c}Q=0.63 \\
P=0.429\end{array}$ & $\begin{aligned} Z & =1.30 \\
P & =0.193\end{aligned}$ \\
\hline \multirow{2}{*}{ Hypertrichosis } & $\begin{array}{l}\text { BIM vs. } \\
\text { LAT }\end{array}$ & 2 & 257 & 0 & 261 & 0 & $\begin{array}{c}0.562(0.002 \\
160.502)\end{array}$ & $\begin{array}{l}Q=12.79 \\
P \leq 0.001\end{array}$ & $\begin{aligned} Z & =0.20 \\
P & =0.842\end{aligned}$ \\
\hline & $\begin{array}{l}\text { BIM vs. } \\
\text { TRA }\end{array}$ & 1 & 131 & 135 & 0 & 0 & $\begin{array}{c}5.152(0.250 \\
106.293)\end{array}$ & N.A. & $\begin{aligned} Z & =1.06 \\
P & =0.288\end{aligned}$ \\
\hline \multirow{3}{*}{ Itching } & $\begin{array}{l}\text { BIM vs. } \\
\text { LAT }\end{array}$ & 1 & 133 & 0 & 136 & 0 & $\begin{array}{c}0.345(0.115 \\
1.031)\end{array}$ & N.A. & $\begin{aligned} Z & =1.90 \\
P & =0.057\end{aligned}$ \\
\hline & $\begin{array}{l}\text { BIM vs. } \\
\text { TRA }\end{array}$ & 2 & 180 & 180 & 0 & 0 & $\begin{array}{c}1.304(0.301 \\
5.641)\end{array}$ & $\begin{array}{c}Q=1.31 \\
P=0.252\end{array}$ & $\begin{aligned} Z & =0.35 \\
P & =0.723\end{aligned}$ \\
\hline & $\begin{array}{l}\text { TAF vs. } \\
\text { LAT }\end{array}$ & 2 & 0 & 0 & 73 & 74 & $\begin{array}{c}0.985(0.349 \\
2.775)\end{array}$ & $\begin{array}{c}Q=0.59 \\
P=0.443\end{array}$ & $\begin{array}{l}Z=0.035 \\
P=0.977\end{array}$ \\
\hline
\end{tabular}

*BIM: bimatoprost, TRA: travoprost, LAT: latanoprost, TAF: tafluprost.

modification of tafluprost is the substitution of the C-15 hydrogen and hydroxyl group with two fluorine atoms [8]. Its metabolite, tafluric acid, is a FP receptor agonist with an affinity for the FP receptor that is 12-fold higher than the affinity of the latanoprost metabolite [33]. Unoprostone is also a PGF $2 \alpha$ analogue. However, due to the docosanoid structure, it has very low affinity for the prostaglandin receptor [34]. Early animal studies suggested that unoprostone can increase uveoscleral pathway outflow by activating the FP receptor [35]. Recent studies found that unoprostone increases TM path outflow through stimulating calciumion- (Ca2+-) activated BK- and CIC-2-type channels $[34,36]$. Currently, the Food and Drug Administration (FDA) has removed the description of this drug as a PGA
[36]. Due to the limitations of existing studies, we did not analyze the responsiveness of unoprostone.

It has been reported that $15 \%$ of patients do not adequately respond to PGAs, which could be partly due to single nucleotide polymorphisms (SNPs) in genes encoding matrix metalloproteinases (MMPs) and PGF2 $\alpha$ receptor (PTGFR) [5]. Sakurai et al. [37] reported the SNPs rs3753380 and rs3766355 in the promoter and intron 1 region of the PTGFR gene in healthy Japanese volunteers can cause downregulation of receptor expression after a short duration of latanoprost treatment, undermining the efficacy. They also found an association between the SNP rs12093097 in the PTGFR gene and the response to latanoprost in patients with glaucoma or OHT [38]. Ussa et al. [14] demonstrated that 
the SNPs rs6686438 and rs10786455 in PTGFR are likely to be related to a positive response to latanoprost, and SNPs rs3753380, rs6672484, and rs11578155 are likely to be related to a negative response to latanoprost in patients with OAG. This study revealed that SNPs in the gene encoding MMP-1 also influence the response to latanoprost. MMPs, which are neutral proteases expressed by TM and uveoscleral tissues, can initiate degradation of ECM and regulate outflow resistance [10]. SNPs also affect the responsiveness to other ocular hypotensive agents in patients with glaucoma. Cytochrome P450 (CYP) 2D6 Arg296Cys is a polymorphic site on the CYP2D6 gene [39]. It has been reported that the Arg296Cys travoprost genotype can increase CYP2D6 activity, enhance timolol metabolism, and thus, reduce timolol therapeutic effects [39].

Several studies have found differences in responsiveness and efficacy of multiple PGAs in OAG/OHT [11, 16, 22, 40], with variations in the molecular structure and mechanism of action of PGAs being a potential reason. Latanoprost and travoprost are synthetic ester prodrugs of natural PGF $2 \alpha$, which necessitate hydrolysis into the active form during the corneal passage $[18,29-31]$. The lack of response to latanoprost may be due, in part, to poor de-esterification of the prodrug to the pharmacologically active free fatty acid [4]. Tafluprost requires to be metabolized by corneal esterase to exert its antihypertensive effect. The difference in corneal permeability could be a factor related to the variation in responsiveness [26]. Bimatoprost, a fatty acidamide, is an amide prodrug of 17-phenyl-PGF2 $\alpha$ [5]. Fatty-acid amides are neutral lipids without the negative charge associated with the carboxylic acid group of fatty acids such as PGF $2 \alpha$, latanoprost, and travoprost. This is an important structural difference of bimatoprost from other PGAs [7]. Bimatoprost can manifest its IOP-lowering ability as a complete prostaglandin-like molecule, without any metabolic conversion $[17,18]$. The hydrolysate of bimatoprost (17-phenyl PGF2 $\alpha$, also named bimatoprost acid) is also an effective FP receptor agonist and can promote outflow facility [7]. Several studies suggested that bimatoprost has unique pharmacological effects. Besides the known prostanoid receptors, it can also act on a dedicated prostamide-sensitive receptor that can partially explain the unique bimatoprost effects compared to other PGAs [5, 7, 10]. Chen et al. [32] reported that bimatoprost can exert its effects on the human $T$ lymphoblast (peripheral blood acute lymphoblastic leukemia, MOlatanoprost-3) cells, while qPCR analysis had proven that MOlatanoprost-3 cells expressed no FP or thromboxane A2 receptors (TP). This result supported the hypothesis that bimatoprost can bind to a unique receptor beyond FP and TP. However, this unique "undefined receptor" has not been cloned in studies thus far [1].

In glaucoma, the increase of ECM in TM can also induce the obstruction of outflow facility. MMPs can degrade the ECM in the TM and uveal region, subsequently skewing the resistance to aqueous humor outflow [29]. Heo et al. [6] found that latanoprost and bimatoprost can effectively upregulate the activities of MMP-1 and MMP-9 in human TM. Furthermore, in the study of Li et al. [10] on immortalized human TM (iHTM) cells, it was found that latanoprost and bimatoprost significantly upregulated the expression of transcription factors c-fos and MMP-9 and downregulated the expression of the tissue inhibitor of metalloproteinase 4 (TIMP-4) simultaneously, consequently promoting the degradation of ECM in the TM and increased outflow facility. This study also revealed that fibronectin mRNA expression was upregulated by latanoprost though downregulated by bimatoprost. Fibronectin can increase the stiffness of the TM, change the resistance to aqueous humor outflow, and finally, increase IOP [41]. Several studies found the fibronectin levels in aqueous humor of glaucomatous patients were nearly seven-fold higher than those of patients with cataracts [42]. The degradation of fibronectin will induce ECM turnover in the TM and inner wall of Schlemm's canal, ultimately affecting the resistance of outflow facility [43]. This can constitute the reason why bimatoprost has enhanced efficacy properties than latanoprost: although both PGAs can activate ECM degeneration, the increased expression of fibronectin might impair the IOP-reducing efficacy of latanoprost. In addition, bimatoprost can significantly downregulate the expression of aquaporin-1 (AQP1) in iHTM [10]. AQPs are 10 AQP families involved in water transportation, which are expressed in multiple organizations [44]. In ocular tissues, AQP1 is expressed in the lens epithelial cells (LECs), ciliary epithelium, and iris. In LECs, the high expression of AQP1 will increase the penetration of the aqueous humor into the lens, resulting in thickening of the lens and, consequently, obstructing the outflow of the aqueous humor [45]. In the iris and ciliary epithelium, AQP1 plays a role in the production of the aqueous humor by transporting water out of the ciliary epithelium [46]. Compared with normal individuals, the IOP of AQP1-knockout mice was significantly decreased [47]. Downregulated AQP1 expression could be one of the reasons why bimatoprost has a higher IOP-reducing efficacy than latanoprost. However, AQP1 is also expressed in the TM and can regulate the volume of trabecular cells [48] and mediate cytoskeleton remodeling [49] and cell migration/ proliferation $[49,50]$ through interactions with $\beta$-catenin. Zhao et al. [46] demonstrated that AQP1 expression was downregulated in TM cells exposed to Endothelin-1 (ET-1), causing glaucomatous changes such as actin fiber reorganization, collagen production, extracellular matrix deposition, and contractility alteration of TM cells, eventually leading to an increase in IOP. The ET-1-induced actin fiber reorganization in human TM cells can be significantly reversed through transfection with an adenoviral vector encoding for full-length AQP1 [46]. Therefore, whether decreasing the expression of AQP-1 can help reduce IOP remains to be explored.

The results of this meta-analysis reveal that bimatoprost responsiveness in patients with OAG/OHT is higher than for latanoprost and travoprost, although this advantage is not statistically significant. Tafluprost responsiveness in patients with OAG/OHT is higher than for latanoprost and travoprost, though this is not statistically significant either. Due to the lack of original data, we did not directly compare the responsiveness of latanoprost and travoprost and bimatoprost and tafluprost in OAG/OHT. Bimatoprost has a higher 
efficacy for reducing IOP than latanoprost. There is no significant variation in the IOP-reducing efficacy of travoprost, latanoprost, and tafluprost. Due to the lack of original data, we did not compare the IOP-reducing efficacy of bimatoprost and travoprost and travoprost and latanoprost, as well as bimatoprost and tafluprost in OAG/OHT. In OAG/OHT patients who do not respond to latanoprost, switching to bimatoprost or travoprost can increase the percentage of patients demonstrating response, with the response to bimatoprost higher than that of travoprost. However, this improvement does not possess statistical significance either. Compared with latanoprost and travoprost, bimatoprost can more effectively lower IOP in LNRs, though there is no significant difference in travoprost and latanoprost efficacy. All four PGA classes exhibited good safety profiles, with no serious, vision-impairing adverse events taking place. The most common adverse event was conjunctival hyperemia. The prevalence of conjunctival hyperemia in patients using bimatoprost or tafluprost is significantly higher than that using latanoprost. The prevalence of conjunctival hyperemia in patients using bimatoprost is also higher than that using travoprost, albeit with no statistical difference. The other adverse events (burning, foreign-body sensation, eyelash hypertrichosis, and itching) demonstrated nonsignificant differences in the three classes of PGAs. The studies conducted by Konstas [22] and Kammer [16] also confirmed that there was no significant difference in the incidence of skin pigmentation among patients using bimatoprost, latanoprost, and travoprost, although the data were insufficient and we did not conduct a meta-analysis.

In the two studies studying the responsiveness of bimatoprost in LNRs, the crossover study conducted by Gandolfi et al. [18], revealed that, among 15 patients who did not respond to latanoprost after 6-8 weeks of treatment, none of the patients exhibited response on continuing latanoprost treatment for one month, while 13 patients exhibited response after converting to bimatoprost treatment for one month. However, Blondeau et al. [17] found that, after one-month treatment of bimatoprost, 13 out of 31 LNRs exhibited response to bimatoprost, with 9 out of 29 converting into responders after continuing latanoprost treatment for one month. The study authors believed that this increase in responsiveness could be partly due to median regression and the Hawthorne effect. The Hawthorne effect is that when subjects know they participate in a study, their behavior may change and demonstrate enhanced patient compliance $[17,51]$. This means that a subgroup of patients might not actually lack in response. Their poor compliance during nonstudy time periods affects the efficacy of the drug. The bimatoprost implant Durysta ${ }^{\mathrm{TM}}$, developed by Allergan (USA), can avoid the impact of patient compliance on efficacy. It can be implanted into the anterior chamber and release bimatoprost slowly and continuously, effectively reducing IOP for 4-6 months after implantation [52]. This therapeutic measure could effectively enhance the patient's response to such drugs. In 2020, bimatoprost implants have been approved in the USA for OAG/OHT treatment [53].
Preservative use is an important factor affecting adverse reactions. All of the four PGA eyedrops studied in this metaanalysis contain preservatives. Benzalkonium chloride (BAK) is a common preservative and is widely used as a component of eyedrops for its antibacterial properties [54]. However, through cellular apoptosis and neurotoxicity, BAK can possibly induce ocular surface diseases (OSDs), leading to several symptoms including dryness, irritation, burning sensations, foreign-body sensations, photophobia, and eye fatigue $[55,56]$. These adverse reactions reduce patient tolerability, leading to decreased patient compliance, and ultimately influence the effectiveness of topical glaucoma therapy [55]. A range of low-toxicity preservatives have been developed to replace BAK, such as SofZia and Polyquaternium-1 (also named polyquad) [57]. Kumar [57] reported that Ocular Surface Disease Index (OSDI) scores were significantly lower in polyquad-preserved travoprost in comparison to BAK-preserved travoprost, with comparable IOP-reducing efficacy. Aihara [54] discovered that switching from BAK-preserved latanoprost to SofZia-preserved travoprost can ameliorate chronic superficial punctate keratitis and there was no significant change in conjunctival hyperemia, tear breakup time (TBUT), or IOP. Despite having low toxicity, such novel preservatives can still cause adverse reactions $[58,59]$. Preservative-free eyedrops may bring enhanced safety. Preservative-free tafluprost (PFtafluprost) is the first preservative-free formulation for a PGA preparation [56, 60]. Hamacher [60] demonstrated that PF-tafluprost had an equivalent IOP-reducing efficacy compared to preservative-containing tafluprost (PCtafluprost) and is well tolerated. Ruangvaravate [56] demonstrated that switching from other preservativecontaining prostaglandins to PC-tafluprost and PF-tafluprost both increased TBUT in glaucoma patients with OSD, while PF-tafluprost had better tear quality versus PCtafluprost. Therefore, PF-tafluprost should be especially beneficial for patients with preexisting OSD. Several studies found that PF-latanoprost had an equivalent IOP-reducing efficacy compared to PC-latanoprost, although it was better tolerated [55, 61]. Preservative-free eyedrops could possibly be the future development tendency for antiglaucoma drugs.

This meta-analysis has several certain limitations. Firstly, the number of included studies is small, which could lead to biased results. Furthermore, this study lacks a direct comparison of the responsiveness and safety of travoprost and latanoprost and bimatoprost and tafluprost, in patients with OAG/OHT. The population demographics included in this meta-analysis included white, black, Hispanic, and Asian. However, we did not conduct subgroup analysis for race due to the limitation of original data. The length of follow-up time of the included studies ranged from one to six months, for it is highly challenging to study the long-term efficacy and responsiveness of the four PGAs in OAG/OHT populations. Finally, there are five PGAs used in clinical treatments, though, due to the lack of original literature, we did not analyze the responsiveness and efficacy of unoprostone. 


\section{Conclusions}

In essence, existing studies highlight that latanoprost, travoprost, bimatoprost, and tafluprost do not have statistically significant differences in responsiveness within the OAG/OHT patient population. OAG/OHT patients who are LNRs cannot get a significant increase in response by switching to travoprost or bimatoprost. The IOP-reducing efficacy of bimatoprost is significantly higher than that of latanoprost. There is no significant difference in the IOPreducing efficacy of travoprost, latanoprost, and tafluprost. All the four PGAs have good safety. The prevalence of conjunctival hyperemia due to bimatoprost or tafluprost is higher than that of latanoprost. Other adverse events show no significant difference between the four drugs.

We hope that additional high-quality and large-sample RCTs can be carried out in the near future to compare the responsiveness and IOP-reducing efficacy of different PGAs in the OAG/OHT patient population.

\section{Data Availability}

The data used to support the findings of this study are available from the corresponding author upon request.

\section{Conflicts of Interest}

The authors declare that they have no conflicts of interest.

\section{Acknowledgments}

This work was supported by the National Natural Science Foundation of China (81970801),National Natural Science Foundation of Hunan (2019JJ4001), and Changsha Science and Technology Bureau Plan Project (kh1801299).

\section{References}

[1] D. Lee, A. V. Mantravadi, and J. S. Myers, "Patient considerations in ocular hypertension: role of bimatoprost ophthalmic solution," Clinical Ophthalmology, vol. 11, pp. 1273-1280, 2017.

[2] S. Guven Yilmaz, C. Degirmenci, Y. E. Karakoyun, E. Yusifov, and H. Ates, "The efficacy and safety of bimatoprost/timolol maleate, latanoprost/timolol maleate, and travoprost/timolol maleate fixed combinations on 24-h IOP," International Ophthalmology, vol. 38, no. 4, pp. 1425-1431, 2018.

[3] T. A. Macky, "Bimatoprost/timolol versus travoprost/timolol fixed combinations in an Egyptian population," Journal of Glaucoma, vol. 23, no. 8, pp. 561-566, 2014.

[4] M. Digiuni, P. Fogagnolo, and L. Rossetti, "A review of the use of latanoprost for glaucoma since its launch," Expert Opinion on Pharmacotherapy, vol. 13, no. 5, pp. 723-745, 2012.

[5] A. Matsou and E. Anastasopoulos, "Investigational drugs targeting prostaglandin receptors for the treatment of glaucoma," Expert Opinion on Investigational Drugs, vol. 27, no. 10 , pp. $777-785,2018$.

[6] J. Y. Heo, Y. H. Ooi, and D. J. Rhee, "Effect of prostaglandin analogs: latanoprost, bimatoprost, and unoprostone on matrix metalloproteinases and their inhibitors in human trabecular meshwork endothelial cells," Experimental Eye Research, vol. 194, Article ID 108019, 2020.
[7] A. H. Krauss and D. F. Woodward, "Update on the mechanism of action of bimatoprost: a review and discussion of new evidence," Survey of Ophthalmology, vol. 49, no. 1, pp. S5-S11, 2004.

[8] J. Ge, X. X. Li, X. H. Sun, X. G. He, and H. Zhang, "[Randomized parallel group study of $0.0015 \%$ tafluprost ophthalmic solution in patients with primary open-angle glaucoma or ocular hypertension (comparison with $0.005 \%$ latanoprost ophthalmic solution)]," China Journal of Ophthalmol, vol. 51, no. 02, pp. 95-102, 2015.

[9] D. F. Woodward, S. F. E. Nilsson, C. B. Toris, A. B. Kharlamb, A. L. Nieves, and A. H.-P. Krauss, "Prostanoid EP4Receptor stimulation produces ocular hypotension by a mechanism that does not appear to involve uveoscleral outflow," Investigative Opthalmology \& Visual Science, vol. 50, no. 7, pp. 3320-3328, 2009.

[10] X. Li, F. He, B. T. Gabelt et al., "Effects of latanoprost and bimatoprost on the expression of molecules relevant to ocular inflow and outflow pathways," PloS One, vol. 11, no. 3, Article ID e0151644, 2016.

[11] N. Choplin, P. Bernstein, A. L. Batoosingh, and S. M. Whitcup, "A randomized, investigator-masked comparison of diurnal responder rates with bimatoprost and latanoprost in the lowering of intraocular pressure," Survey of Ophthalmology, vol. 49, no. 1, pp. S19-S25, 2004.

[12] Y. Ikeda, K. Mori, T. Ishibashi, S. Naruse, N. Nakajima, and S. Kinoshita, "Latanoprost nonresponders with open-angle glaucoma in the Japanese population," Japanese Journal of Ophthalmology, vol. 50, no. 2, pp. 153-157, 2006.

[13] A. Martínez García and R. Pérez García, "[Efficacy of latanoprost monotherapy treatment in primary open-angle glaucoma]," Archivos de la Sociedad Espanola de Oftalmologia, vol. 77, no. 5, pp. 269-274, 2002.

[14] F. Ussa, I. Fernandez, M. Brion et al., "Association between SNPs of metalloproteinases and prostaglandin F $2 \alpha$ receptor genes and latanoprost response in open-angle glaucoma," Ophthalmology, vol. 122, no. 5, pp. 1040-1048, 2015.

[15] W. J. Scherer, "A retrospective review of non-responders to latanoprost," Journal of Ocular Pharmacology and Therapeutics, vol. 18, no. 3, pp. 287-291, 2002.

[16] J. A. Kammer, B. Katzman, S. L. Ackerman, and D. A. Hollander, "Efficacy and tolerability of bimatoprost versus travoprost in patients previously on latanoprost: a 3month, randomised, masked-evaluator, multicentre study," British Journal of Ophthalmology, vol. 94, no. 1, pp. 74-79, 2010.

[17] P. Blondeau, M. Hamid, and Z. Ghalie, "Prospective randomized clinical trial on the effects of latanoprost, travoprost and bimatoprost on latanoprost non-responders," Journal Français d'Ophtalmologie, vol. 42, no. 8, pp. 894-899, 2019.

[18] S. A. Gandolfi and L. Cimino, "Effect of bimatoprost on patients with primary open-angle glaucoma or ocular hypertension who are nonresponders to latanoprost," Ophthalmology, vol. 110, no. 3, pp. 609-614, 2003.

[19] D. F. Woodward, A. H.-P. Krauss, J. Chen et al., "The pharmacology of bimatoprost (Lumigan)," Survey of Ophthalmology, vol. 45, no. 4, pp. S337-S345, 2001.

[20] C. Lavia, L. Dallorto, M. Maule, M. Ceccarelli, and A. M. Fea, "Minimally-invasive glaucoma surgeries (MIGS) for open angle glaucoma: a systematic review and meta-analysis," $\mathrm{PloS}$ One, vol. 12, no. 8, Article ID e0183142, 2017.

[21] R. S. Noecker, M. S. Dirks, N. T. Choplin, P. Bernstein, A. L. Batoosingh, and S. M. Whitcup, "A six-month 
randomized clinical trial comparing the intraocular pressurelowering efficacy of bimatoprost and latanoprost in patients with ocular hypertension or glaucoma," American Journal of Ophthalmology, vol. 135, no. 1, pp. 55-63, 2003.

[22] A. G. P. Konstas, G. Holló, M. Irkec et al., "Diurnal IOP control with bimatoprost versus latanoprost in exfoliative glaucoma: a crossover, observer-masked, three-centre study," British Journal of Ophthalmology, vol. 91, no. 6, pp. 757-760, 2007.

[23] R. J. Noecker, M. L. Earl, T. K. Mundorf, S. M. Silverstein, and M. P. Phillips, "Comparing bimatoprost and travoprost in black Americans," Current Medical Research and Opinion, vol. 22, no. 11, pp. 2175-2180, 2006.

[24] Y. Kuwayama and S. Komemushi, "[Phase III confifirmatory study of $0.0015 \%$ DE-085 (taflfluprost) ophthalmic solution as compared to $0.005 \%$ latanoprost ophthalmic solution in patients with open angle glaucoma or ocular hypertension]," Atarashii Ganka (Journal of the Eye), vol. 25, no. 11, pp. 1595-1602, 2008.

[25] C. E. Traverso, A. Ropo, M. Papadia, and H. Uusitalo, "A phase II study on the duration and stability of the intraocular pressure-lowering effect and tolerability of Tafluprost compared with latanoprost," Journal of Ocular Pharmacology and Therapeutics, vol. 26, no. 1, pp. 97-104, 2010.

[26] T. Mizoguchi, M. Ozaki, K. Unoki, Y. Dake, T. Eto, and M. Arai, "A randomized crossover study comparing tafluprost $0.0015 \%$ with travoprost $0.004 \%$ in patients with normaltension glaucoma [corrected]," Clinical Ophthalmology, vol. 6, pp. 1579-1584, 2012.

[27] T. Li, K. Lindsley, B. Rouse et al., "Comparative effectiveness of first-line medications for primary open-angle glaucoma," Ophthalmology, vol. 123, no. 1, pp. 129-140, 2016.

[28] M. V. Boland, A.-M. Ervin, D. S. Friedman et al., "Comparative effectiveness of treatments for open-angle glaucoma: a systematic review for the U. S. Preventive Services Task Force," Annals of Internal Medicine, vol. 158, no. 4, pp. 271-279, 2013.

[29] C. B. Toris, B. T. Gabelt, and P. L. Kaufman, "Update on the mechanism of action of topical prostaglandins for intraocular pressure reduction," Survey of Ophthalmology, vol. 53, no. 1, pp. S107-S120, 2008.

[30] L. Quaranta, I. Riva, I. Floriani, M. Centofanti, A.-G. Katsanos, and A. G. Konstas, "Safety and efficacy of travoprost solution for the treatment of elevated intraocular pressure," Clinical Ophthalmology, vol. 9, pp. 633-643, 2015.

[31] C. M. Perry, J. K. McGavin, C. R. Culy, and T. Ibbotson, "Latanoprost," Drugs \& Aging, vol. 20, no. 8, pp. 597-630, 2003.

[32] J. Chen, R. T. Lu, R. Lai et al., "Bimatoprost-induced calcium signaling in human T-cells does not involve prostanoid FP or TP receptors," Current Eye Research, vol. 34, no. 3, pp. 184-195, 2009.

[33] G. M. Keating, "Tafluprost ophthalmic solution 0.0015\%: A review in glaucoma and ocular hypertension," Clinical Drug Investigation, vol. 36, no. 6, pp. 499-508, 2016.

[34] N. V. Harms and C. B. Toris, "Current status of unoprostone for the management of glaucoma and the future of its use in the treatment of retinal disease," Expert Opinion on Pharmacotherapy, vol. 14, no. 1, pp. 105-113, 2013.

[35] J. Serle, S. M. Podos, Y. Kitazawa, and R. F. Wang, "A comparative study of latanoprost (Xalatan) and isopropyl unoprostone (Rescula) in normal and glaucomatous monkey eyes," Japanese Journal of Ophthalmology, vol. 42, no. 2, pp. 95-100, 1998.
[36] D. S. Fung and J. T. Whitson, "An evidence-based review of unoprostone isopropyl ophthalmic solution $0.15 \%$ for glaucoma: place in therapy," Clinical Ophthalmology (Auckland, N. Z.), vol. 8, pp. 543-554, 2014.

[37] M. Sakurai, T. Higashide, M. Takahashi, and K. Sugiyama, "Association between genetic polymorphisms of the prostaglandin F2 $\alpha$ receptor gene and response to latanoprost," Ophthalmology, vol. 114, no. 6, pp. 1039-1045, 2007.

[38] M. Sakurai, T. Higashide, S. Ohkubo, H. Takeda, and K. Sugiyama, "Association between genetic polymorphisms of the prostaglandin $\mathrm{F} 2 \alpha$ receptor gene, and response to latanoprost in patients with glaucoma and ocular hypertension," British Journal of Ophthalmology, vol. 98, no. 4, pp. 469-473, 2014.

[39] Y. Yang, K. Wu, H. Yuan, and M. Yu, "Cytochrome oxidase 2D6 gene polymorphism in primary open-angle glaucoma with various effects to ophthalmic timolol," Journal of Ocular Pharmacology and Therapeutics, vol. 25, no. 2, pp. 163-172, 2009.

[40] M. Centofanti, F. Oddone, M. Vetrugno et al., "Efficacy of the fixed combinations of bimatoprost or latanoprost plus timolol in patients uncontrolled with prostaglandin monotherapy: a multicenter, randomized, investigator-masked, clinical study," European Journal of Ophthalmology, vol. 19, no. 1, pp. 66-71, 2009.

[41] C. Wang, L. Li, and Z. Liu, "Experimental research on the relationship between the stiffness and the expressions of fibronectin proteins and adaptor proteins of rat trabecular meshwork cells," BMC Ophthalmology, vol. 17, no. 1, p. 268, 2017.

[42] J. A. Faralli, M. S. Filla, and D. M. Peters, "Role of fibronectin in primary open angle glaucoma," Cells, vol. 8, no. 12, 2019.

[43] J. Ueda, K. Wentz-Hunter, and B. Y. Yue, "Distribution of myocilin and extracellular matrix components in the juxtacanalicular tissue of human eyes," Investigative Ophthalmology \& Visual Science, vol. 43, no. 4, pp. 1068-1076, 2002.

[44] Y. Fu, J. Zhu, Y. Zhang, Z. Liu, H. Su, and J. Kong, "Vitamin D regulates the expressions of AQP-1 and AQP-4 in mice kidneys," Biomed Research International, vol. 2019, Article ID 3027036, , 2019.

[45] L. Cheng, B. Long, X. X. Guo et al., "Increased aquaporin-1 levels in lens epithelial cells with primary angle-closure glaucoma," International Journal of Ophthalmology, vol. 10, no. 7, pp. 1101-1105, 2017.

[46] Y. Zhao, H. Zhu, Y. Yang et al., “AQP1 suppression by ATF4 triggers trabecular meshwork tissue remodelling in ET-1induced POAG," Journal of Cellular and Molecular Medicine, vol. 24, no. 6, pp. 3469-3480, 2020.

[47] D. Zhang, L. Vetrivel, and A. S. Verkman, "Aquaporin deletion in mice reduces intraocular pressure and aqueous fluid production," Journal of General Physiology, vol. 119, no. 6, pp. 561-569, 2002.

[48] C. H. Mitchell, J. C. Fleischhauer, W. D. Stamer, K. PetersonYantorno, and M. M. Civan, "Human trabecular meshwork cell volume regulation," American Journal of Physiology-Cell Physiology, vol. 283, no. 1, pp. C315-C326, 2002.

[49] R. Madonna, Y.-J. Geng, H. Shelat, P. Ferdinandy, and R. De Caterina, "High glucose-induced hyperosmolarity impacts proliferation, cytoskeleton remodeling and migration of human induced pluripotent stem cells via aquaporin-1," Biochimica et Biophysica Acta (BBA)-Molecular Basis of Disease, vol. 1842, no. 11, pp. 2266-2275, 2014.

[50] X. Yun, H. Jiang, N. Lai, J. Wang, and L. A. Shimoda, "Aquaporin 1-mediated changes in pulmonary arterial 
smooth muscle cell migration and proliferation involve $\beta$-catenin," American Journal of Physiology-Lung Cellular and Molecular Physiology, vol. 313, no. 5, pp. L889-L898, 2017.

[51] B. M. Morberg, A. S. Malling, B. R. Jensen, O. Gredal, L. Wermuth, and P. Bech, "The Hawthorne effect as a preplacebo expectation in Parkinsons disease patients participating in a randomized placebo-controlled clinical study," Nordic Journal of Psychiatry, vol. 72, no. 6, pp. 442-446, 2018.

[52] M. Shirley, "Bimatoprost implant: first approval," Drugs \& Aging, vol. 37, no. 6, pp. 457-462, 2020.

[53] P. E. Miller and J. S. Eaton, "Medical anti-glaucoma therapy: beyond the drop," Veterinary Ophthalmology, vol. 24, no. 1, pp. 2-15, 2021.

[54] M. Aihara, Y. Ikeda, S. Mizoue, Y. Arakaki, N. Kita, and S. Kobayashi, "Effect of switching to travoprost preserved with SofZia in glaucoma patients with chronic superficial punctate keratitis while receiving BAK-preserved latanoprost," Journal of Glaucoma, vol. 25, no. 6, pp. e610-e614, 2016.

[55] P. Harasymowycz, C. Hutnik, J. F. Rouland et al., "Preserved versus preservative-free latanoprost for the treatment of glaucoma and ocular hypertension: a post hoc pooled analysis," Advances in Therapy, vol. 2021, 2021.

[56] N. Ruangvaravate, K. Choojun, B. Srikulsasitorn, J. Chokboonpiem, D. Asanatong, and S. Trakanwitthayarak, "Ocular surface changes after switching from other prostaglandins to tafluprost and preservative-free tafluprost in glaucoma patients," Clinical Ophthalmology, vol. 14, pp. 3109-3119, 2020.

[57] S. Kumar, T. Singh, P. Ichhpujani, and S. Vohra, "Ocular surface disease with BAK preserved travoprost and Polyquaternium 1(polyquad) preserved travoprost," Romanian Journal of Ophthalmology, vol. 63, no. 3, pp. 249-256, 2019.

[58] T. Kanamoto, Y. Kiuchi, M. Tanito et al., "Comparison of the toxicity profile of benzalkonium chloride-preserved tafluprost and SofZia-preserved travoprost applied to the ocular surface," Journal of Ocular Pharmacology and Therapeutics, vol. 31, no. 3, pp. 156-164, 2015.

[59] J. H. Peace, P. Ahlberg, M. Wagner, J. M. Lim, D. Wirta, and J. D. Branch, "Polyquaternium-1-Preserved travoprost $0.003 \%$ or benzalkonium chloride-preserved travoprost $0.004 \%$ for glaucoma and ocular hypertension," American Journal of Ophthalmology, vol. 160, no. 2, pp. 266-e1, 2015.

[60] T. Hamacher, J. Airaksinen, V. Saarela, M. J. Liinamaa, U. Richter, and A. Ropo, "Efficacy and safety levels of preserved and preservative-free tafluprost are equivalent in patients with glaucoma or ocular hypertension: results from a pharmacodynamics analysis," Acta Ophthalmologica, vol. 86, pp. 14-19, 2008.

[61] M. Misiuk-Hojlo, M. Pomorska, M. Mulak et al., "The RELIEF study: tolerability and efficacy of preservative-free latanoprost in the treatment of glaucoma or ocular hypertension," European Journal of Ophthalmology, vol. 29, no. 2, pp. 210-215, 2019. 